\title{
PRIMARY METASTATIC ATRIAL SARCOMA: CASE REPORT
}

\author{
Carramiñana Domínguez A ${ }^{1}$, Escudero Teixidó A ${ }^{1}$, Ibáñez Esteve $C^{1}$, Cámara Rosell M², Teis Soley A ${ }^{3}$, Canet Capeta J \\ ${ }^{1}$ Department of Anaesthesia and Intensive Care. Germans Trias \& Pujol University Hospital, Badalona, Spain \\ ${ }^{2}$ Department of Cardiovascular Surgery. Germans Trias \& Pujol University Hospital, Badalona, Spain \\ ${ }^{3}$ Department of Cardiology. Germans Trias \& Pujol University Hospital, Badalona, Spain
}

\section{BACKGROUND}

Primary cardiac tumours are rare diseases, with an estimated prevalence of 0.02 to $0.3 \%$ depending on the databases. Around $75 \%$ of them are benign (nearly $50 \%$ myxomas), while the remaining quarter is malignant. Within the latter, sarcomas are the most common subtype, but there is limited data available. On the other hand, secondary heart metastatic tumours are 50 times more common than primary ones ${ }^{(1,2)}$. Heart tumour symptoms can be obstructive, embolic, constitutional or caused by local invasion (2).

Recent imaging advances have improved the diagnostic process, which includes echocardiography (TTE or TEE), computed tomography (CT) and Magnetic Resonance (MR). In addition, pathological diagnosis can be obtained through biopsy or surgical excision. However, it is very difficult to systematically classify these tumours ${ }^{(1)}$ and the best treatment approach for cardiac sarcoma is still under discussion. The role of adjuvant chemotherapy (QT), radiation therapy (RT) and cardiac transplantation remains controversial, with consensus just about better prognosis with surgical resection ${ }^{(3)}$.

\section{CASE REPORT}

We discuss the case of a 55-year-old lady without previous history presented with a four month history of back pain with progressive loss of strength and sensibility in her lower limbs. Conventional X-ray and MRI showed D6 fracture with vertebral collapse and medullary compression. The patient underwent medullary decompression with D6 resection and posterior RT. Because of dyspnea and lower extremity edema a TTE was performed. It showed 63x50mm left atrial mass infiltrating both superior and inferior left pulmonary veins associating mitral stenosis due to tumour protrusion, conditioning heart failure with severe dyspnea.

Fluid and neoadjuvant QT administration were limited because of 2 episodes of pulmonary edema solved with nitroglycerin (GTN) and furosemide.

After discussion, cardiac surgery via a median sternotomy was performed with partially tumour resection (see images below). The patient was extubated $9 \mathrm{~h}$ after in the ICU and was discharged home seventeen days after surgery without relevant postoperative complications. Histology confirmed undifferentiated pleomorphic sarcoma with bone metastasis (D6).

During five months after the operation the patient received adjuvant QT with Adriamycin. Unfortunately, six months after the operation she died because of a respiratory infection.

\section{DISCUSSION AND LEARNING POINTS}

Cardiac sarcomas are definitely uncommon diseases. Like our patient, up to $80 \%$ of patients have evidence of metastasis at the time of diagnosis. Complete surgical excision is the only therapy that has been proved effective for symptoms and outcome but despite of this fact, mean survival is less than a year.

The most common cause of death is heart failure or mass recurrence ${ }^{(3)}$. In our patient, palliative resection was performed because of the impossibility of administrating neoadjuvant QT due to severe dyspnea, with significant postoperative symptomatic improvement. Adjuvant QT and fluid were administered five months postoperatively with no cardiac compromise. More evidence is required to identify the best therapeutic approaches for those difficult diseases in order to improve patients outcome.
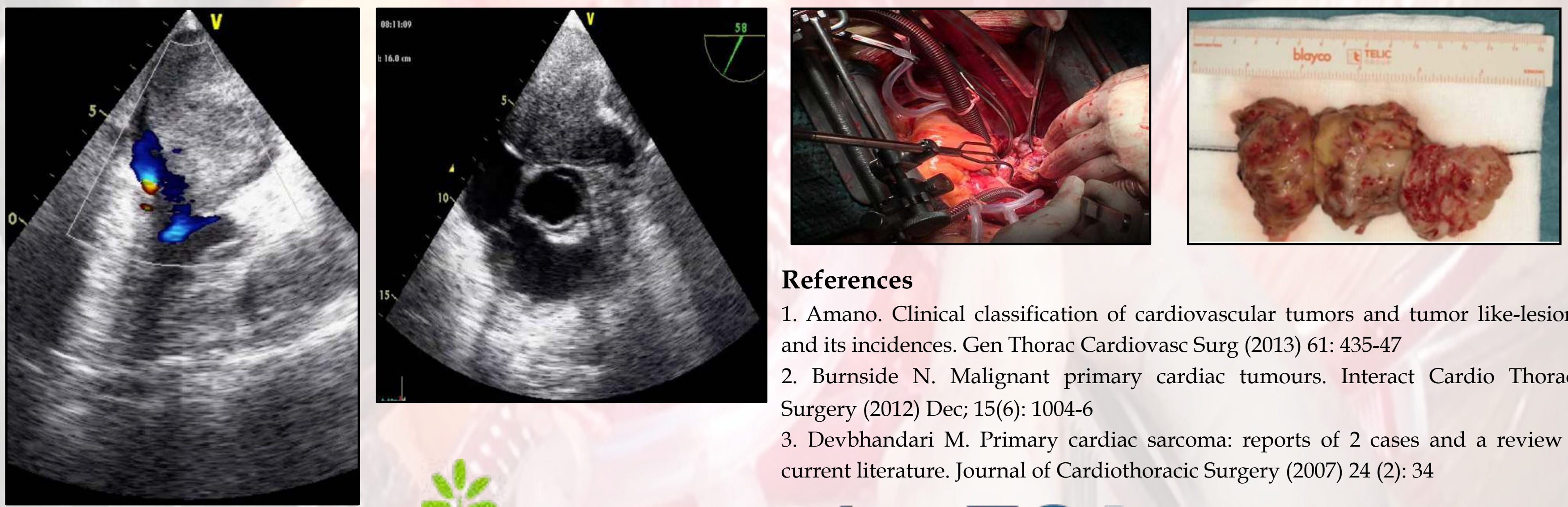

\section{References}

1. Amano. Clinical classification of cardiovascular tumors and tumor like-lesions, and its incidences. Gen Thorac Cardiovasc Surg (2013) 61: 435-47

2. Burnside N. Malignant primary cardiac tumours. Interact Cardio Thoracic Surgery (2012) Dec; 15(6): 1004-6

3. Devbhandari M. Primary cardiac sarcoma: reports of 2 cases and a review of current literature. Journal of Cardiothoracic Surgery (2007) 24 (2): 34 